\title{
DNA is a new acceptor of PARP3 protein
}

E. Belousova ${ }^{1 *}$, A. Ishchenko², O. Lavrik ${ }^{1}$

${ }^{1}$ Institute of Chemical Biology and Fundamental Medicine SB RAS, Novosibirsk, Russia

${ }^{2}$ UMR8200 - CNRS Paris Saclay, Gustave Roussy, 94805 Villejuif, France

*e-mail:rina@niboch.nsc.ru

Key words: PARP3, mono(ADP-ribosyl)ation of DNA, DNA repair, NHEJ

Motivation and Aim: Poly(ADP-ribose)polymerases, PARPs, represent a protein family that is involves in a number of cellular processes such as DNA repair, genomic stability, and programmed cell death. Through the whole family that includes 17 participants with very different structures and cellular functions, only three proteins - PARP1, PARP2 and PARP3, possess DNA-dependent catalytic activity on attaching of ADP-ribose from $\mathrm{NAD+}+$ molecule to the acceptors [1]. Interestingly, that PARP1 and PARP2 catalyzed synthesis of long stretch of poly(ADP-ribose)polymers, whereas the most data are consider that PARP3 is a mono(ADP-ribose)transferase.

For a long time, amino acid residue of the protein substrate such as aspartate, glutamate and lysine, have been exclusive acceptor for PARP-enzymes. However, it was recently discovered by Talhaoui et al. that DNA-dependent PARP1 and PARP2 can also modify DNA $[2,3]$. Here, we demonstrate that DNA-dependent PARP3 can modify DNA and form a specific primed structure for further use by the repair proteins.

Methods and Algorithms: In this study, we discovered the capacity of recombinant human PARP3 to modify partial DNA duplexes and investigated in detail the nature of the DNA modification and its processing by the DNA repair proteins.

Results: Present study demonstrates that DNA-dependent PARP3 can modify DNA and that this ADP-ribosylated DNA could be ligated to double-stranded DNA by DNA ligases. Moreover, ADP-ribosylated DNA could serve as a primed DNA substrate for PAR chain elongation by the purified proteins PARP1 and PARP2 as well as by cell-free extracts.

Conclusion: Based on the obtained results we propose that ADP-ribose modification of DNA can be involved in cellular pathways that are important for cell survival in the process of double-strand break formation. Moreover, our results suggest that the different PARPs might act in concert to ensure the efficiency of some cellular processes. Acknowledgements: Supported by the Russian Science Foundation 14-24-0038.

\section{References}

1. Vyas et al. (2014). Family-wide analysis of poly(ADP-ribose) polymerase activity. Nat Commun. 5:4426.

2. Talhaoui et al. (2016) Poly(ADP-ribose) polymerases covalently modify strand break termini in DNA fragments in vitro. Nucleic Acids Res. 44(19):9279-9295.

3. Munnur and Ahel (2017) Reversible mono-ADP-ribosylation of DNA breaks. FEBS J. 284(23):40024016. 